Revue d'histoire de l'Amérique française

REVUE D.HISTOIRE DE L'AMÉRIQUE FRANÇAISE

\title{
Mutations de la Confédération des travailleurs catholiques du Canada (1940-1960)
}

\section{Jacques Rouillard}

Volume 34, numéro 3, décembre 1980

URI : https://id.erudit.org/iderudit/303879ar

DOI : https://doi.org/10.7202/303879ar

Aller au sommaire du numéro

Éditeur(s)

Institut d'histoire de l'Amérique française

ISSN

0035-2357 (imprimé)

1492-1383 (numérique)

Découvrir la revue

Citer cet article

Rouillard, J. (1980). Mutations de la Confédération des travailleurs catholiques du Canada (1940-1960). Revue d'histoire de l'Amérique française, 34(3), 377-405. https://doi.org/10.7202/303879ar d'utilisation que vous pouvez consulter en ligne.

https://apropos.erudit.org/fr/usagers/politique-dutilisation/ 


\title{
MUTATIONS DE LA CONFÉDÉRATION DES TRAVAILLEURS CATHOLIQUES DU CANADA (1940-1960)
}

\author{
JACQUES ROUILLARD \\ Département d'histoire \\ Université de Montréal
}

Il n'y a rien de très nouveau à affirmer que la CTCC a subi de profondes mutations depuis la guerre. Les ouvrages d'histoire l'ont répété et les dirigeants de la centrale ont clamé bien haut qu'il $\mathrm{y}$ avait une nette brisure entre eux et leurs prédécesseurs ${ }^{1}$. Pour Jean Marchand, secrétaire général de la centrale de 1947 à 1961, puis président de 1961 à 1965 , la CTCC, à ses débuts, était nettement «nationaliste, confessionnelle, procorporatiste et les aumôniers y jouaient un rôle prédominant». "Souvent, écrit-il, l'action catholique prenait le pas sur l'action professionnelle et proprement syndicale. ${ }^{2}$ Le leadership syndical des années 1950 avait la nette conviction de rompre avec le passé, de sortir la centrale d'un ténébreux Moyen âge.

Nul ouvrage n'a fait davantage pour accréditer cette thèse que le volume édité par Pierre-Elliott Trudeau en 1956 sur la grève de l'amiante (1949). Pour un des collaborateurs, Réginald Boisvert, la CTCC, pendant près de trente ans de vie, ne s'était pas précisément signalée par son dynamisme; le regain de vigueur qu'elle avait manifesté à l'occasion de la grève reflétait le rejet du «conservatisme social» et l'adoption d'une «doctrine dynamique, pleinement consciente de l'évolution de notre milieu» ${ }^{3}$.Poussant encore

1 Comme exemple d'ouvrages ayant adopté ce point de vue, signalons: CSN-CEQ, Histoire du mouvement ouvrier du Québec (1825-1976) (Montréal, CSN-CEQ, 1979), 124-131; Louis-Marie Tremblay, Le syndicalisme québécois: idéologies de la CSN et de la FTQ (1940-1970) (Montréal, PUM, 1972), 23-27; Hélène David, «L'état des rapports de classe au Québec de 1945 à 1967», Sociologie et sociétés (novembre 1975): 33-65; Roch Denis, Luttes de classes et question nationale au Québec (1948-1968) (Montréal, Presses socialistes internationales, 1979), 128-134.

2 Jean Marchand, "La CSN a quarante ans», Relations industrielles, 16, 4 (octobre 1961): 471.

3 Pierre-Elliott Trudeau, La Grève de l'amiante (Montréal, Ed. du jour), 357 et 359.

RHAF, vol. 34, no 3, décembre 1980

[377] 
plus loin leur réflexion, certains collaborateurs du volume ont fait, des changements subis par la centrale, l'agent de régénération de la société québécoise. Selon Trudeau, Boisvert et Jean-Charles Falardeau, les travailleurs de la CTCC ont montré l'irréalisme de la doctrine sociale de l'Église pour lui substituer des objectifs plus conformes à leurs besoins véritables. Plus pragmatiques, moins doctrinaires, "parce que leur vie se déroule au niveau de l'immédiat», les travailleurs, écrit Trudeau, ont été les premiers à comprendre le sens de l'industrialisation opérée durant la Guerre et à faire éclater les contradictions entre l'idéologie et la réalité industrielle ${ }^{4}$. Pour celui-ci, qui écarte le «messianisme prolétarien» et la "mystique révolutionnaire», ce renouveau est porteur "de liberté et de justice» dans la société québécoise ${ }^{5}$. Estimant que les travailleurs constituaient «une force puissante d'assainissement des lois et des moeurs démocratiques», il avait décelé dans le mouvement ouvrier les éléments de la réforme pour laquelle il luttait depuis longtemps, soit un redressement de la démocratie libérale ${ }^{6}$. Il interprétait les mulations subies pâr la CTCC comme des signes avant-coureurs de changements profonds dans la société, changements que concrétisera la Révolution tranquille.

Il n'est pas dans notre intention au cours des pages qui suivent de vérifier l'hypothèse qui lie la Révolution tranquille au mouvement ouvrier; plus modestement, nous nous proposons de faire ressortir les transformations qui ont conduit la centrale catholique à démontrer un plus grand militantisme et à redéfinir son orientation idéologique. Trois thèmes retiendront notre attention: l'évolution idéologique, la déconfessionnalisation et l'action syndicale.

\section{A) ÉVOLUTION IDÉOLOGIQUE:}

\section{DU CORPORATISME Ȧ L'HUMANISME DÉMOCRATIQUE}

\section{Le corporatisme}

Depuis sa fondation, l'objectif ultime de la CTCC était l'instauration de la corporation professionnelle au Québec. En organisant les professions en corporation, la centrale catholique pensait

4 Ibid., 89.

5 Ibid., 404.

6 Cité libre (décembre 1952): 66, dans M. Behields, Prelude to Revolution: An analysis of Reform Ideology in Quebec, Ph.D. (histoire) (York University, 1978), 422. 
corriger les abus du capitalisme et restaurer la paix sociale. La corporation professionnelle, telle que la concevait la centrale, regroupait des représentants des travailleurs et des employeurs qui veilleraient aux intérêts communs de la profession. Des questions comme les salaires, les conditions de travail, l'apprentissage, le contrôle de la production et même le partage des profits devaient faire l'objet de discussion en comité mixte par les membres de la corporation ${ }^{7}$. Dans un premier temps, la corporation devait couvrir le Québec par secteur industriel, et être coiffée par la suite d'une organisation intercorporative, toujours composée de travailleurs et de patrons, qui verrait aux intérêts généraux des professions. Grâce à ces structures, on pensait mettre un terme aux conflits sociaux et instaurer une collaboration permanente entre l'élément patronal et ouvrier.

Il est important d'ajouter que ce système ne pouvait fonctionner que dans la mesure où un esprit nouveau animait les acteurs sociaux. Aux rapports conflictuels dans l'entreprise, devait succéder un esprit de coopération fondé sur la justice et la charité. L'Église se chargeait de la tâche de transformer les mentalités pour faire disparaître l'égoïsme du patronat et la méfiance des travailleurs. L'instauration du corporatisme ne comportait pas seulement la mise en place d'une structure juridique, elle conviait à une transformation morale des individus.

Même si la CTCC a eu comme objectif jusqu'à la guerre l'établissement du corporatisme, nous avons montré ailleurs que très tôt, dès les années 1920, ses syndicats négociaient avec le patronat dans un esprit de rapport de forces ${ }^{8}$. Jusqu'à ce que les employeurs changent leur mentalité, on se croyait justifié de défendre du mieux possible les intérêts professionnels des travailleurs, en n'hésitant pas à recourir à la grève si elle s'avérait nécessaire. Dans leur pratique syndicale quotidienne, les syndicats internationaux et catholiques en sont venus à se ressembler étrangement. La négociation de conventions collectives avantageuses requérait la plus grande part de leur énergie et même le programme législatif de la CTCC s'est graduellement aligné sur celui des syndicats internationaux. En fait, la CTCC n'avait pas d'autre choix si elle entendait

7 Voir à ce sujet: J. Rouillard, Les syndicats nationaux au Québec de 1900 à 1930 (Québec, PUL, 1979), 199, 200, 228, 229, 310, 311; La Vie syndicale, août 1934, 6, janvier 1940, 1, décembre 1940, 5; Semaines Sociales du Canada, L'Organisation professionnelle (Montréal, ESP, 1936), 392p.

8 Nous avons développé cette thèse dans notre volume, op. cit., 240-250. 
protéger les intérêts matériels de ses membres; si elle avait agi différemment, elle aurait vu ses adeptes l'abandonner. Il s'est établi ainsi un divorce entre le discours corporatiste de la centrale et ses pratiques syndicales. Pendant que les leaders prêchaient la concorde et l'harmonie avec le patronat, les syndicats négociaient selon un rapport de forces.

Mais il n'était pas encore question pour la centrale dans les années 1920 et 1930 de remettre en cause l'idéologie qui avait présidé à sa fondation. Au contraire, lorsque après la crise, les milieux intellectuels catholiques désireux de restaurer l'ordre social remirent à l'honneur l'étude du corporatisme, la CTCC précisa la conception qu'elle se faisait de son fonctionnement. Ainsi, en 1939, elle chargea le bureau confédéral d'élaborer un programme de réformes corporatistes. Remis l'année suivante, le rapport préconisait que la corporation professionnelle reposât sur une base syndicale, que son caractère chrétien fût assuré et que les syndicats internationaux en fussent exclus ${ }^{9}$. Tel que défini par le bureau confédéral, le corporatisme devenait un moyen de promotion du syndicalisme catholique puisqu'on refusait aux non-syndiqués et aux internationaux le droit d'être représentés dans les corporations ${ }^{10}$.

Discuté et amendé, le programme du bureau confédéral fut finalement adopté par le congrès de 1942. Comme dans le projet initial, la résolution insistait pour édifier la corporation à partir d'organisations syndicales; quant au syndicalisme international, s'il n'était pas explicitement exclu, on hypothéquait sa présence en exigeant de la corporation qu'elle fût revêtue d'un caractère chrétien et que le syndicat partie de sa formation fût incorporé? ${ }^{11}$. Quoiqu'encore assez vague dans son application, le plan révèle néanmoins le souci de la CTCC d'écarter les projets alors en vogue qui ne faisaient pas reposer la corporation sur le mouvement syndical. La centrale voyait aussi dans son établissement le moyen d'éliminer les syndicats internationaux qui, de toute façon, n'auraient jamais collaboré à sa formation car ils dénonçaient le corporatisme comme une "dictature fasciste sur les organisations ouvrières»" ${ }^{12}$. Malheureusement pour les théoriciens du syndicalisme catholique,

9 La Vie syndicale (décembre 1940): 5.

10 Jean Sexton, La CTCC-CSN: du corporatisme à la réforme de l'entreprise, maîtrise en relations industrielles (Université Laval, 1979), 49.

11 CTCC, Procès-verbal du congrès (1942): 136-138.

12 Le Monde ouvrier, 7 août 1937, 4. 
le programme de la CTCC eut peu de suite, car la guerre mobilisait toutes les énergies de la centrale.

Après le conflit, les déclarations procorporatistes se firent plus rares; on préférait même substituer à ce terme des expressions comme «organisation professionnelle» ou même «démocratisation de l'économie»" ${ }^{13}$. La mise en sourdine du projet corporatiste s'explique pour une raison bien simple: ce projet était associé beaucoup trop intimement au fascisme de sorte qu'il devenait gênant d'en réclamer l'application, surtout au sortir d'une guerre contre des régimes qui en avaient fait la base de leur organisation sociale. D'ailleurs, l'application qu'en avaient faite ces pays (Italie, France, Espagne, Portugal, etc.) n'avait rien de réjouissant pour les tenants du syndicalisme libre ${ }^{14}$. C'est ainsi que la CTCC répudia graduellement le corporatisme, modèle de relations industrielles qui avait été à l'origine de sa fondation. Une question vient aussitôt à l'esprit: par quoi l'a-t-elle remplacé?

\section{La réforme de l'entreprise}

Pour quelques années, elle substitua au corporatisme l'idée de la réforme de l'entreprise mise au point après la guerre par des penseurs catholiques français. Reprise au Québec par de jeunes membres du clergé, l'idée fit son chemin parmi la nouvelle génération de dirigeants à la tête de la CTCC. La diffusion de cette nouvelle interprétation de la doctrine sociale de l'Église fut le fait de la Commission sacerdotale d'études sociales qui organisa en 1947 des journées d'études pour les aumôniers et qui en publia le compte rendu sous la forme d'une brochure, La Participation des travailleurs à la vie de l'entreprise ${ }^{15}$. Leurs travaux inspirèrent la lettre pastorale des évêques du Québec de 1950 (Le problème ouvrier en regard de la doctrine sociale de l'Église) dont quelques paragra-

13 J. Sexton, op. cit., 54.

14 En France, la politique du gouvernement de Vichy consistera en 1940 à dissoudre les confédérations syndicales afin de faciliter, disait-on, l'intégration des travailleurs dans le corporatisme d'industrie. Cette décision achèvera de discréditer le corporatisme aux yeux de la Confédération française des travailleurs chrétiens (CFTC) La C.F.D.T. (Paris, Seuil, 1971), 11 et 34).

15 Commission sacerdotale d'études sociales, La participation des travailleurs à la vie de l'entreprise, Compte rendu des journées sacerdotales d'études sociales de 1947 (Montréal, 1949), $100 \mathrm{p}$. 
phes font référence à la nécessité de transformer la structure de l'entreprise ${ }^{16}$.

Sans répudier encore formellement le corporatisme, ces nouveaux théoriciens de la doctrine sociale de l'Église lui reprochaient son globalisme et prétendaient plutôt que la réforme de la société doit d'abord passer par la cellule de base du système économique, l'entreprise ${ }^{17}$. Ils proposaient donc une réforme de l'entreprise qui fasse une part à la participation des travailleurs à ses bénéfices, à sa gestion et à sa propriété. Pour eux, le droit de propriété du patron sur son usine n'est pas absolu car l'entreprise possède un caractère social. Puisque la production, les profits et la croissance de l'entreprise résultent de l'apport du capital et du travail, ils déduisaient un droit limité des travailleurs à la participation aux bénéfices, à la gestion et à la propriété de leur entreprise ${ }^{18}$. Même si, dans la pratique, ils atténuaient beaucoup la portée de leurs principes, il n'en reste pas moins que leurs prémisses entraient en conflit direct avec la notion de propriété telle qu'elle prévalait en Âmérique du Nơrủ.

Le projet de réforme de l'entreprise fut repris par la CTCC qui, à son congrès de 1948 , en entreprenait l'étude et la diffusion parmi ses membres ${ }^{19}$. La nouvelle déclaration de principes adoptée en préambule de sa constitution en 1951 le fixe comme objectif souhaitable. «Dans l'entreprise, peut-on lire, les travailleurs doivent être considérés comme des coopérateurs participant à une oeuvre commune. Ils doivent s'y sentir intégrés et participer à sa gestion et à ses bénéfices ${ }^{20}$. Toutefois, l'idée dans sa forme originale fut pratiquement abandonnée deux ans plus tard, face aux résistances qu'elle rencontrait dans à peu près tous les milieux.

Parce qu'elle restreignait le droit de gérance, le monde patronal y était particulièrement allergique. La question divisa le groupe patronal catholique, l'Association professionnelle des Industriels (API), fondée en 1943 comme complément des syndicats en vue de

16 Évêques du Québec. Le problème ouvrier en regard de la doctrine sociale de l'Église (Montréal, Bellarmin, 1950), 26 et 27. Sur les circonstances ayant entouré la rédaction de cette lettre, on peut lire avec profit Gérard Dion, «La Petite histoire d'un grand document», Perspectives sociales, 25, 2 (mars-avril 1970): 42-47.

17 Jean Sexton, op. cit., 69-133.

18 Commission sacerdotale..., op.cit., 13-45. Voir aussi à ce sujet, les articles de G. Dion, P.-E. Bolté et M. Clément, dans le Bulletin des relations industrielles (1947-1948) parus sous le titre de «Réforme de la structure de l'entreprise».

19 CTCC, Procès-verbal du congrès (1948): 225.

20 Ibid., 1951, 217. 
l'établissement de la corporation. L'un de ses fondateurs, le Père Bouvier, fut même forcé de donner sa démission comme aumônier à cause de ses critiques à l'endroit de la réforme de l'entreprise ${ }^{21}$. Quoiqu'on y fasse très peu allusion dans le volume publié sur la grève de l'amiante sous la direction de Pierre-Elliott Trudeau, cette question fut au centre du conflit, selon la partie patronale ${ }^{22}$.

Pour le président de la Canadian Johns Manville, Lewis $\mathrm{H}$. Brown, l'origine de la grève ne concernait pas les salaires ou les conditions de travail, mais relevait d'une tentative de la part des chefs ouvriers de "s'arroger les droits de la Direction»" ${ }^{23}$. Les demandes syndicales comportaient, en effet, une clause inacceptable selon la Compagnie, soit celle où le syndicat réclamait son approbation aux promotions, mesures disciplinaires et à certaines questions reliées à la production, aux méthodes de travail et aux taux de rémunération ${ }^{24}$. L'idée de cogestion mise de l'avant par les tenants du projet de réforme de l'entreprise avait inspiré ces demandes syndicales ${ }^{25}$. La Compagnie qui en avait fait une question de principe les considérait comme une atteinte au droit de gérance. Il se peut que ces objections n'aient été qu'un paravent pour cacher les véritables motifs d'opposition de la Compagnie, mais il nous apparaît que cette question a joué, à tout le moins dans la première phase du conflit, un rôle capital dans le raidissement de la partie patronale. Dans l'entente finale qui mit fin à la grève, celle-ci insistait pour que le syndicat «reconnaisse le droit de propriété» et «consente à incorporer dans le contrat une clause dite des droits de la direction ${ }^{26}$.

Dans d'autres conflits, la CTCC rencontra aussi une résistance aussi opiniâtre du patronat à l'idée de cogestion, en particulier lors de la grève du textile en 1947 (Lachute) et de l'aluminium en 1951 (Shawinigan) ${ }^{27}$. Cette résistance ne fut pas étrangère à l'aban-

21 Yvan Sénecal, L'Association professionnelle des industriels, thèse de maîtrise en relations industrielles (Université de Montréal, 1954), 88-89.

22 Pierre-Elliott Trudeau, op. cit., 173, 192, 385.

23 L.H. Brown, La grève d'Asbestos, Brochure distribuée par la compagnie Johns Manville (1949), 9.

24 Ibid., 8. Selon la version syndicale, on réclamait uniquement de la direction qu'elle consulte le syndicat dans tous les cas de promotion, de transferts et de congédiements (P.-E. Trudeau, op. cit., 214).

25 A. Charpentier, Les Mémoires d'Alfred Charpentier (Québec, PUL, 1971), 330-

333; Jean Sexton, op. cit., $112 \mathrm{~s}$.

26 P.-E. Trudeau, op. cit., 230.

27 CTCC, Procès-verbal du congrès (1951): 36. 
don par la CTCC de ses idées dans les années 1950. Très avancé pour l'époque, le projet de réforme de l'entreprise tranchait nettement avec les demandes syndicales habituellement formulées en Amérique du Nord. La compagnie américaine Canadian Johns Manville qui s'en était rapidement aperçue demandait au gouvernement de tracer «une ligne de démarcation logique entre ce qui constitue les négociations collectives et ce qui constitue le droit du patronat ${ }^{28}$.

\section{Un humanisme démocratique}

Après avoir abandonné le corporatisme et connu des déboires avec son projet de réforme de l'entreprise, la CTCC évolua au niveau idéologique vers ce que nous appellerions un humanisme démocratique. La déclaration de principes de la centrale élaborée en 1951 et en 1960 loge à cette enseigne idéologique qui prévaudra jusqu'au milieu des années 1960, alors qu'elle radicalisera son discours.

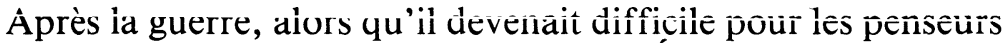
catholiques de prêcher le corporatisme, l'Église se réconciliera dans une certaine mesure avec le libéralisme et la démocratie. Il est significatif de constater que les Semaines sociales, qui reflètent les préoccupations des penseurs sociaux catholiques, ont consacré deux sessions à ces thèmes en 1942 et $1945^{29}$. Les idées libérales que peu de temps auparavant on rejetait parce qu'issues de la Révolution française ne suscitaient plus la même réprobation. Des penseurs catholiques français, J. Maritain, E. Mounier, le Père Delos, et des revues comme Esprit, Témoignage Chrétien et Économie et Humanisme ont fortement influencé nos penseurs sociaux. Plusieurs de ces catholiques «de gauche», comme on les appellera plus tard, puisaient leur inspiration dans le personnalisme, doctrine qui se propose de faire un heureux mariage entre l'autonomie et la responsabilité sociale des individus.

Rejetant l'individualisme parce qu'il referme l'individu sur lui-même, le personnalisme vise à ce que la personne s'ouvre à autrui et s'insère dans une communauté naturelle. Cet aspect de la

28 L.H. Brown, op. cit. 16

29 La Démocratie (1942); Liberté et libertés (1945). Il est étonnant de constater qu'il n'existe pas d'études sur l'influence du personnalisme au Canada français, si ce n'est, dans un esprit polémique, la brochure de Robert Rumilly, L'infiltration gauchiste au Canada français (Montréal, l'auteur, 1956, 147p.). Il a très bien décelé en quoi ce nouveau courant rompait avec la pensée traditionnelle de l'Église. 
pensée personnaliste n'a rien de très neuf par rapport à l'enseignement traditionnel de l'Église. Là où ce courant ouvre de nouvelles perspectives, c'est lorsqu'il propose comme «affirmation centrale» l'existence de personnes libres et créatrices ${ }^{30}$. Bien que Mounier se défende bien de proposer «la liberté du libéralisme», il pose néanmoins comme postulat à sa pensée l'autonomie des personnes et valorise la liberté de choix, ce qui était étranger à la philosophie traditionnelle de l'Église pour qui la liberté, loin d'être une valeur, découlait de la faiblesse de la nature humaine ${ }^{31}$. Ces orientations nouvelles ont influencé la jeune génération de clercs et de laïcs québécois d'après-guerre, qui ne manifestaient plus les mêmes craintes envers les idéaux de liberté et de démocratie. Il y eut chez eux un net effort pour élargir l'autonomie du temporel et rapprocher l'Église de la démocratie politique et sociale.

Ces tendances qui se reflètent dans les discours des leaders syndicaux et des aumôniers de la CTCC ont conduit la centrale à reviser, en 1951, pour la première fois de son histoire, le préambule de sa constitution.

Les auteurs du texte sont animés d'un souffle démocratique tout à fait absent de la première version de la constitution, celle de 1921. On y proclame la foi de la CTCC dans la «vraie démocratie politique», système qui garantit le mieux la liberté des citoyens, et on y affirme l'égalité de tous les êtres humains et l'opposition de la centrale à «tout traitement injuste à cause de la langue, de la nationalité, de la race, du sexe et de la religion ${ }^{32}$. C'est un appui aux droits fondamentaux de l'homme que proclame la centrale; elle l'applique même à la religion, se plaçant en contradiction avec ellemême puisqu'elle est une centrale confessionnelle. La constitution revisée de 1960 accentue encore plus l'appui de la CTCC aux valeurs de liberté et de démocratie ${ }^{33}$. C'est pourquoi nous qualifions d'humanisme démocratique, l'évolution idéologique de la centrale après la guerre.

Cet humanisme ressort du désir de la centrale d'humaniser l'économie, c'est-à-dire d'orienter la production des biens d'abord

30 E. Mounier, Le personnalisme (Paris, PUF, 1950), 6.

31 E. Mounier, Qu'est ce que le personnalisme? (Paris, Seuil, 1946), 97.

32 CTCC, Procès-verbal du congrès (1951): 215. Dans la même veine, elle forme même en 1954 un comité chargé de préparer un projet de déclaration des droits fondamentaux de la personne humaine qui devrait être inscrite dans la constitution canadienne (CTCC, Procès-verbal du congrès (1954): 144).

33 CTCC, Procès-verbal du congrès (1960): 131-138. 
et avant tout vers la satisfaction des besoins humains. La valeur «travail», selon la centrale, doit passer avant le capital car la personne doit primer sur l'accumulation de biens. Comme le capitalisme libéral basé sur la notion de profit maximum déprécie les valeurs humaines et spirituelles, la centrale le répudie, quoiqu'elle reconnaisse la légitimité du droit de propriété privée. En réalité, ce qu'elle condamne, c'est moins le capitalisme comme tel que ses abus, qui doivent être corrigés en intégrant la participation des travailleurs à la vie de l'entreprise et en faisant appel à l'État pour surveiller l'activité économique ${ }^{34}$. L'idée de participation à la vie de l'entreprise qui avait débouché peu après la guerre sur la cogestion, la copropriété et la participation aux bénéfices restera présente parmi les objectifs de la centrale, quoique plus vague dans ses applications concrètes. L'entreprise est encore définie comme une communauté de personnes qui appelle pour cette raison à la participation des travailleurs ${ }^{35}$. On ne doit pas s'étonner que la CTCC critique le capitalisme libéral car elle l'avait fait constamment depuis sa fondation; ce qu'elle a modifié après la guerre, ce sont ses propositions de rechange.

L'humanisme démocratique de la CTCC se manifeste aussi à travers un thème nouveau apparu en 1958, la planification économique $^{36}$. L'humanisation du système économique commande, selon elle, que l'État planifie son développement. L'anarchie qui a jusque-là présidé à son expansion a engendré l'insécurité et l'inégalité, causes de malaises sociaux permanents. L'intervention indicative de l'État par l'élaboration d'un plan avec la collaboration des agents socio-économiques corrigera certains abus du système et orientera davantage l'économie vers la satisfaction des besoins réels des personnes. Ici encore, la CTCC subissait l'influence des débats qui animaient sa pendante française, la CFTC, qui se montrera ouverte à cette réforme à partir de $1957^{37}$. Pour les deux centrales, la planification avait pour objectif d'humaniser et de démocratiser le système économique.

Pendant les vingt années qui ont suivi la guerre, la CTCC a traversé une période de transformations idéologiques profondes. Depuis sa fondation, elle s'était efforcée d'offrir aux travailleurs une troisième voie entre le capitalisme et le socialisme. Ce fut tout

34 CTCC, Procès-verbal du congrès (1951): 216-217.

35 J. Marchand, Rapport du président, CTCC, Procès-verbal (1961): 11 et 23.

36 Louis-Marie Tremblay, op. cit., 78-80.

37 G. Adam, La C.F.T.C., 1940-1958 (Paris, Armand Colin, 1964), 286 et 293. 
d'abord le corporatisme, puis après la guerre, la réforme de l'entreprise et un humanisme démocratique. Dans un de nos ouvrages, nous avons montré que le patronat qui n'a pas mordu à l'idéal corporatiste était le véritable responsable de l'échec de cette idéologie $^{38}$. Dans les pages qui précèdent, nous avons fait ressortir que ce même patronat a donné le coup de mort à l'idée de réforme de l'entreprise car elle ne rencontrait pas les canons de sa définition de la libre entreprise. L'humanisme démocratique qui lui a succédé comportait lui aussi un idéal difficilement conciliable avec le capitalisme nord-américain. Aussi la CTCC-CSN abandonnerat-elle cette option dans les années 1960 pour s'orienter vers le socialisme démocratique, un modèle de société dont elle accentuera la critique du capitalisme.

\section{B) LA DÉCONFESSIONNALISATION DE LA CTCC}

Parallèlement à des changements d'orientation idéologique, la CTCC a graduellement perdu, à partir de la guerre, son caractère confessionnel. À son origine, quatre caractéristiques, selon nous, en faisaient une centrale catholique: l'addition de l'épithète "catholique» à son appellation, la présence d'un aumônier, l'adhésion à la doctrine sociale de l'Église et l'obligation d'être catholique pour obtenir tous les droits de membre actif. Graduellement, la CTCC va se départir de chacun de ces traits pour devenir une centrale non-confessionnelle ouverte à tous les travailleurs. C'est ce cheminement que nous allons retracer au cours des paragraphes suivants.

Lors des réunions qui allaient conduire à la fondation de syndicats catholiques, il avait été prévu qu'on réserverait aux seuls catholiques le privilège d'être membres actifs des syndicats. Les non-catholiques n'étaient pas formellement exclus, mais ils étaient admis à titre de membres adjoints seulement, sans droit de vote, ni possibilité d'occuper une fonction de direction ${ }^{39}$. En fait, peu de syndicats comptèrent des membres adjoints et l'habitude se développa rapidement dans une ville aussi cosmopolite que Montréal de ne pas faire en pratique de discrimination à l'endroit des non-catholiques. Leur nombre infime ne justifiait pas qu'on s'alarmât de leur présence. D'ailleurs, les non-catholiques n'étaient

38 J. Rouillard, op. cit., $311 \mathrm{~s}$.

39 Ibid, 231. 
guère intéressés à joindre des syndicats qui leur accordaient, du moins officiellement, un statut d'infériorité.

Ce n'est que pendant la guerre que la CTCC réalisa que cette discrimination représentait un obstacle au recrutement d'adhérents. Comme la guerre créait un contexte favorable à la syndicalisation, elle ressentait vivement le fait que les centrales rivales progressaient à un rythme supérieur au sien. En 1942, une enquête ordonnée par le cardinal Villeneuve à ce propos suggérait la suppression de toute discrimination dans le recrutement des noncatholiques ${ }^{40}$.

L'année suivante, d'autres événements extérieurs à la centrale forcèrent la CTCC à revoir sa position à l'égard des non-catholiques. En effet, la mise en force graduelle au Canada, dans les industries de guerre, de l'équivalent de la loi américaine Wagner plaça la CTCC dans une situation délicate. Sous l'inspiration de cette loi, le gouvernement canadien passa plusieurs arrêtés en conseil touchant les relations de travail dans les industries de guerre $\left(75 \%\right.$ de toutes les industries) ${ }^{41}$. Les commissions gouvernementales chargées d'appliquer ces décrets reconnurent, comme aux États-Unis, le monopole de représentation au syndicat majoritaire dans une entreprise. Là où deux ou plusieurs syndicats se disputaient l'adhésion des travailleurs, un vote d'allégeance était pris pour déterminer le syndicat qui négocierait la prochaine convention collective. Ainsi, la loi amenait-elle les syndicats de la CTCC à représenter des non-catholiques, auxquels pourtant ils accordaient un statut d'infériorité.

Ce problème surgit avec beaucoup d'acuité en 1943 dans l'industrie du papier de la région du Saguenay. La compagnie Price Brothers avait accordé l'atelier fermé à deux syndicats internationaux, devenus par la suite minoritaires dans les quatre usines de la Compagnie. Mais celle-ci refusait de négocier avec les syndicats catholiques parce qu'ils ne représentaient pas les ouvriers de métier essentiels au fonctionnement de l'usine et que leur reconnaissance signifiait l'obligation pour les non-catholiques d'appartenir à une organisation confessionnelle ${ }^{42}$. Le conflit qui dégénéra en une

40 A. Charpentier, op. cit., 401.

41 Ibid., 285.

42 Québec, Commission Prévost chargée de faire enquête sur certains différends survenus aux usines de Price Brothers \& Company, Limited, et de Lake St. John Power \& Paper Company, Limited (août 1943), 1-10. 
grève conduisit le gouvernement à mettre sur pied une Commission royale d'enquête qui recommanda l'annulation de la clause d'atelier fermé et la reconnaissance des syndicats catholiques majoritaires $^{43}$. Le gouvernement se rendit aux désirs de la Commission mais en obligeant aussi les quatre syndicats de la CTCC de supprimer le mot catholique de leur appellation et d'accorder l'égalité de droits et privilèges à tous leurs membres ${ }^{44}$.

Quelques mois plus tard, le président de la CTCC, A. Charpentier, invitait les délégués au congrès de la centrale à ouvrir le mouvement "dans un large esprit de fraternité ouvrière» ${ }^{45}$. La suggestion créa des tiraillements mais se traduisit quand même dans une résolution qui exigeait des membres de la centrale, non pas qu'ils soient catholiques, mais, selon une formulation plus vague, «qu'ils conforment leurs paroles et leurs actes de syndiqués aux principes directeurs de la $\mathrm{CTCC} \gg{ }^{46}$. Ainsi, à condition d'adhérer aux principes de la doctrine sociale de l'Église, un non-catholique pouvait être membre de plein droit de la centrale. La CTCC éliminait donc un premier critère de confessionnalité en donnant à tout adhérent un statut d'égalité peu importe son appartenance religieuse.

Un pas plus important vers la déconfessionnalisation fut franchi seize ans plus tard, en 1960, alors que la CTCC supprima deux autres de ses caractères confessionnels: l'épithète catholique de son appellation et toute référence directe à la doctrine sociale de l'Eglise. Dans sa déclaration de principes, la phrase où elle disait «s'inspirer dans ses principes et son action de la doctrine sociale de l'Église») fut remplacée par une autre où elle reconnaissait n'adhérer qu'à des principes chrétiens, dans sa pensée et son action ${ }^{47}$. Entre Confédération des Syndicats chrétiens et Confédération des Syndicats nationaux (CSN), la majorité des délégués se rallièrent au deuxième nom, éliminant ainsi tout caractère religieux de l'appellation de la centrale.

Encore une fois, à la fin des années 1950, la CTCC traversait une période de recrutement difficile, dont on espérait sortir en se montrant plus accueillant pour les non-catholiques. En milieu cos-

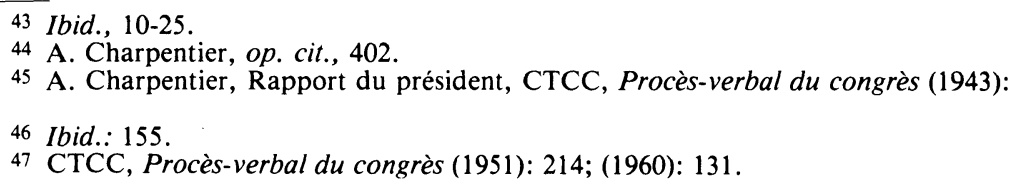


mopolite comme Montréal, la CTCC avait peine à percer dans les grandes entreprises à cause de son caractère confessionnel. Lors de la tenue d'un scrutin dans les unités de négociation pour choisir le syndicat majoritaire, la CTCC faisait face à un sérieux handicap car il fallait décompter au départ le vote de la plupart des noncatholiques. En régime de monopole de représentation syndicale tel qu'il existait en Amérique du Nord, la confessionnalité imposait à la CTCC une contrainte majeure. «Ou bien, disait le rapport du comité exécutif, la CTCC accepte le défi et fonde un grand nombre de syndicats majoritaires, ou bien elle se condamne ellemême à la stagnation et sans doute à une éventuelle disparition.» ${ }^{48}$ Ainsi présenté, le dilemne ne fut pas difficile à résoudre pour les délégués au congrès de 1960, surtout que depuis quatre ans la centrale étudiait la question et qu'elle avait reçu l'assurance que l'épiscopat ne s'objecterait pas en principe aux modifications du statut confessionnel ${ }^{49}$.

L'abandon par la centrale de deux de ses plus importants traits confessionneis suscita plus de remoús pârimi les aumôniers que parmi les syndiqués. Cinq anciens aumôniers publièrent en juillet 1959 une brochure d'une cinquantaine de pages accusant les dirigeants de la CTCC de vouloir laïciser la centrale, c'est-à-dire de lui faire perdre les caractères qui avaient été à l'origine de sa fondation ${ }^{50}$. Ils n'avaient pas tort sur ce point, mais l'assemblée des évêques appelée à se prononcer peu après jugea que le nouveau projet «reflétait suffisamment la doctrine sociale de l'Église» pour être acceptable. Les évêques terminaient leur lettre en affirmant que c'était la responsabilité de la centrale d'effectuer des modifications à la constitution si elle le jugeait nécessaire ${ }^{51}$. En somme, l'épiscopat reconnaissait l'entière autonomie de la CTCC, signe révélateur d'une évolution considérable.

Cette évolution reflétait les changements survenus dans la société québécoise depuis l'après-guerre. Un nouveau courant de pensée est apparu dans l'Église, chez certains clercs et laïs qui voulaient établir une distinction beaucoup plus claire entre l'action temporelle et le spirituel et redonner aux laïcs catholiques une

48 Ibid. (1960): 103.

49 Ibid., 110.

50 Considérations d'anciens aumôniers fondateurs de syndicats catholiques (Québec, juillet $1959,55 \mathrm{p}$.)

51 Lettre de C.-O. Garant au secrétaire de la CTCC, 6 août 1960, CTCC, Procèsverbal du congrès (1960): 130. 
autonomie plus grande dans l'action sociale. Contrairement à leurs prédécesseurs, ils pensaient que des domaines comme le syndicalisme ou le mouvement coopératif étaient des activités temporelles qui ne relevaient pas directement du pouvoir spirituel. Non pas qu'ils rejetaient que des principes chrétiens dussent guider l'action sociale, mais leur solution devait provenir du jugement des fidèles et non de directives précises de la hiérarchie. Les laïcs catholiques engagés dans l'action sociale, qui revendiquaient une plus grande part de responsabilités dans l'orientation de leur mouvement, ne prisaient guère de se voir soumis à des règles d'action précises. Ainsi les aumôniers jouèrent-ils un rôle beaucoup plus discret dans les syndicats de la CTCC après la guerre. Lorsque les leaders de la CTCC décidèrent de s'affranchir de la tutelle de l'Église en 1960, l'évolution des esprits était trop avancée pour que les évêques puissent y faire obstacle.

Après le congrès de 1960, seule la présence d'un aumônier conférait encore à la centrale un semblant de confessionnalité. Il va sans dire qu'il joua un rôle de plus en plus effacé si bien que lorsqu'il remit sa démission en 1971, le Conseil confédéral ne demanda pas son remplacement ${ }^{52}$. Les syndicats et les organismes régionaux étaient cependant libres de maintenir un service d'aumônier, pratique que plusieurs avaient déjà abandonnée ou abandonnèrent par la suite. Ce dernier épisode de la déconfessionnalisation passa inaperçu tellement la CSN s'était éloignée des principes qui avaient présidé à sa fondation.

Pour l'essentiel, le processus de déconfessionnalisation de la CTCC s'était effectué en deux temps, en 1943 et surtout en 1960. Deux raisons majeures nous semblent l'expliquer, la crainte d'une stagnation de ses effectifs et un désir d'autonomie des dirigeants de la CTCC dans l'action temporelle. Ces changements s'étaient faits sans trop de heurts, signe qu'ils reflétaient l'évolution de l'esprit de ses membres, et signe aussi que cette évolution était déjà largement accomplie dans les corps affiliés à la centrale.

\section{C) L'ACTION SYNDICALE}

Il est généralement admis que la CTCC s'est montrée beaucoup plus militante après la guerre: elle aurait soutenu de nombreuses grèves, fait une critique vigoureuse du patronat et serait

52 CTCC, Procès-verbal du congrès (1972): 186. 
devenue un des principaux centres d'opposition au régime Duplessis. La source principale de ce revirement tiendrait au renouveau du leadership syndical entrepris à la CTCC après la guerre. Selon A. Charpentier, ancien président, en 1949, plus de la moitié des directeurs du Bureau confédéral étaient des nouveaux venus ${ }^{53}$. Plusieurs de ces jeunes étaient fraîchement sortis de la Faculté des sciences sociales de l'Université Laval, où ils s'étaient frottés au nouveau courant d'interprétation de la doctrine sociale de l'Église.

Comme nous l'avons fait remarquer en introduction, ils avaient l'impression d'insuffler à la centrale un dynamisme qu'elle n'avait jamais possédé. Ils voyaient dans la CTCC d'avant-guerre plus un mouvement d'action catholique qu'une organisation de défense professionnelle. Soumise à l'influence du clergé, la CTCC, pensaient-ils, recherchait davantage la collaboration du patronat qu'elle ne se souciait de la défense des intérêts de ses membres. D'après eux, le recours à la grève était tenu en suspicion et les membres trop militants, écartés de ses rangs. Selon un calcul fait par un des auteurs d'une histoire de la CSN publiée en $19 \overline{9} 6 \hat{3}$, Jean Francoeur, à peine neuf grèves sur 507 déclarées dans la province auraient été l'oeuvre de la CTCC entre 1915 et 1936. «Il faut bien admettre, conclut-il, que la grève était presque complètement hors des perspectives d'action de cette centrale syndicale. $\rangle^{54}$

Dans un historique des syndicats nationaux de 1900 à 1930, nous avons remis en question cette image de la CTCC pendant ses dix premières années d'existence. Après une analyse exhaustive, nous concluions que la centrale catholique se distinguait assez peu des syndicats internationaux dans ses pratiques syndicales. Les deux mouvements qui avaient un programme législatif assez similaire, travaillaient à la signature de bonnes conventions collectives de travail; ils recherchaient l'atelier fermé et utilisaient en guise de moyens de pression l'étiquette syndicale et la grève ${ }^{55}$. Contrairement à l'affirmation de Jean Francoeur, nous avons recensé 32 grèves conduites par des syndicats catholiques uniquement entre 1920 et 1930 . Celles-ci représentaient $13 \%$ des grèves survenues au Québec pendant ces dix années, alors que la CTCC groupait environ $27 \%$ des syndiqués québécois ${ }^{56}$. Parmi ces grèves, certaines

53 A. Charpentier, op. cit., 341.

54 En collaboration, En grève! l'histoire de la CSN et de ses luttes de 1937 à 1963 (Montréal, Ed. du jour, 1963), 94.

55 J. Rouillard, op. cit., 249.

56 Ibid., 243. 
ont eu beaucoup de retentissement: la grève des policiers et pompiers de Québec en 1921, celle des ouvrières de la manufacture Eddy à Hull en 1924, et surtout la grève des travailleurs de la chaussure de Québec en 1926 (88 677 jours ouvrables perdus).

Puisque la fréquence du déclenchement d'une grève est un bon indice d'évaluation du militantisme syndical, nous avons poursuivi notre recherche pour les années ultérieures et constaté qu'entre 1931 et 1940, la CTCC était responsable d'au moins 21 grèves, soit $9 \%$ des grèves déclenchées pendant ces années ${ }^{57}$. Les syndicats catholiques comptaient alors pour environ le tiers des syndiqués québécois. Avant de conclure trop rapidement au peu d'agressivité de la CTCC, il faut tenir compte que plusieurs des conflits étaient déclenchés par des non-syndiqués et que la CTCC recruta surtout des travailleurs ayant un faible pouvoir de marchandage: cuir, textile, vêtement, services ${ }^{58}$. Nous admettons que les syndicats internationaux montraient plus d'empressement à se servir de la grève, mais la différence n'est pas si grande. Deux des grèves soutenues par la CTCC sont parmi les plus importantes de la période en terme de jours ouvrables perdus, celles de la Dominion Textile (200 000) et des chantiers maritimes de Sorel (31 400) en 1937. Ces faits contredisent l'image jusqu'ici reçue de complaisance de la CTCC à l'égard du patronat. Son idéologie corporatiste ne l'a pas empêchée de se porter à la défense des travailleurs quand le besoin s'en est fait sentir. "Les syndicats catholiques, écrivait aux évêques l'aumônier général, l'abbé $M$. Fortin, en 1925 , ne sont pas des associations pieuses ou des confréries; ils n'ont pas non plus comme objectif d'exhorter les ouvriers à pratiquer le renoncement, la patience et la résignation (...), mais leur raison d'être, c'est de veiller, comme tout syndicalisme, aux intérêts de la classe ouvrière. $\gg^{59}$

Si telle était la conduite des syndicats catholiques d'avantguerre, y a-t-il eu alors des changements dans les pratiques syndicales de la centrale après le conflit mondial? Le revirement ne serait-il pas plus idéologique (abandon du corporatisme) qu'inscrit dans le concret de l'action syndicale? Nous allons vérifier cette

\footnotetext{
58 Canada, Ministère du travail, Labour Organization in Canada (1931): 56-62; (1940): 20-37.

59 Maxime Fortin, Mémoire sur le syndicalisme au Canada (Montréal, Beauchemin, 1927), 17.
} $1931-1940$

57 Canada, Ministère du travail, Organisation des travailleurs au Canada, 
hypothèse en analysant les rapports de la CTCC avec le patronat, les centrales rivales et le gouvernement provincial.

\section{Le patronat}

Lorsqu'on dépouille l'organe de la CTC, Le Travail, on est surpris de constater que le ton des articles a changé dans les années 1950; les rédacteurs du journal se montrent plus agressifs envers le patronat. Depuis sa fondation, la CTCC dénonçait les abus du capitalisme, mais elle le fit après la guerre avec une virulence qu'on ne lui connaissait pas. Et l'idée de vouloir dépasser le capitalisme par la réforme de l'entreprise n'est pas étrangère à ses critiques, du moins au début de la période. Il faut dire aussi que le patronat ne l'avait pas épargnée, l'ayant acculée à quelques grèves longues et dures, en particulier celles d'Asbestos (1949), de Louiseville (1952), de Dupuis Frères (1952) et de Radio-Canada (1959). En décembre 1952, au plus fort de la grève de Louiseville, le comité plénier de la centrale décida même du principe d'une grève générale $^{60}$. Bien que les dirigeants $n^{\prime}$ aient pas donné suite à cette résolution, le geste est révélateur de leur état d'esprit.

Comme pour les périodes antérieures, nous avons calculé le pourcentage de grèves soutenues par la CTCC. Pour les années où nous disposons de statistiques, soit entre 1952 et $1960,34 \%$ des conflits survenus au Québec ont impliqué des syndicats affiliés à la CTCC $(99 / 287)^{61}$. Mis en relation avec le pourcentage de syndiqués que détenait la centrale au Québec, environ 30\%, les données démontrent bien la combativité des syndicats catholiques. Compte tenu que des non-syndiqués sont responsables de plusieurs grèves, la CTCC possède plus que sa part de conflits.

Désireuse de se donner les moyens de lutter plus efficacement, la centrale approuva en 1949 la constitution d'un fonds spécial de défense professionnelle afin d'aider une fédération ou un syndicat dans «des circonstances extraordinaires» ${ }^{62}$. Certaines fédérations professionnelles avaient déjà constitué un fonds de grève; les autres étaient invitées à se doter d'un tel fonds le plus rapidement possible. La CTCC commença à percevoir une cotisation (10 sous par mois) pour son fonds spécial en 1951, cotisation qu'elle aug-

60 En collaboration, En grève!..., op. cit., 164-176.

61 Canada, Ministère du Travail, Grèves et lock-out au Canada, 1952-1960; Québec, Ministère du Travail, Rapport du ministère du Travail du Québec, 1952, 1960; H. David, op. cit., 63. Je remercie mon assistant de recherche, Réjean Myre, qui a compilé ces statistiques. 62 CTCC, Procès-verbal du congrès (1949): 189-191. 
menta par la suite régulièrement ${ }^{63}$. Le patronat et le gouvernement posaient assez d'obstacles à l'exercice du droit de grève, soutenaiton, pour que la centrale se donne les moyens d'assurer le minimum de protection aux travailleurs ${ }^{64}$.

Cette recrudescence du militantisme syndical valut aux syndicats catholiques la désapprobation de plusieurs milieux dont, aussi surprenant que cela puisse paraître, celui de certains chefs ouvriers internationaux importants qui reprirent à leur compte l'accusation que certaines sections de la CTCC possédaient une mentalité révolutionnaire apparentée à celle des communistes ${ }^{65}$. En présentant son rapport au congrès de 1953 , le directeur du service d'organisation de la centrale, R. Harmegnies, avouait que les syndicats catholiques ne bénéficiaient plus de l'appui qu'ils recevaient auparavant d'autres milieux sociaux. «Dans plusieurs cas, soulignait-il, cet appui s'est transformé en hostilité déclarée». ${ }^{66}$ Face à cette opposition accrue, les délégués réunis en congrès en 1953, loin de mettre la pédale douce, votèrent à l'unanimité une résolution affirmant que la CTCC ne priverait jamais ses membres de l'exercice du droit de grève et qu'elle ne se ferait jamais «la complice de l'exploitation des travailleurs ${ }^{67}$. Et effectivement, elle démontra la même combativité dans les années subséquentes.

\section{Les centrales syndicales}

Si sa fougue lui aliéna la Fédération provinciale des Travailleurs du Québec (FPTQ), la centrale québécoise qui regroupait les syndicats internationaux affiliés à la Fédération américaine du Travail (FAT) ${ }^{68}$, la CTCC se trouva des alliés du côté de la Fédération des Unions industrielles du Québec (FUIQ) qui réunissait les syndicats du Congrès des Organisations industrielles (COI) et de l'Alliance des Professeurs de Montréal, en butte à l'hostilité de la CECM et du gouvernement Duplessis. Née assez tard, en 1952, la FUIQ a tout de suite senti des affinités avec la CTCC. Les deux recrutaient un bassin de travailleurs assez identiques, les travailleurs industriels, les deux voulaient dépasser le syndicalisme d'affaires, les deux souhaitaient une intervention accrue de l'État et, enfin, les

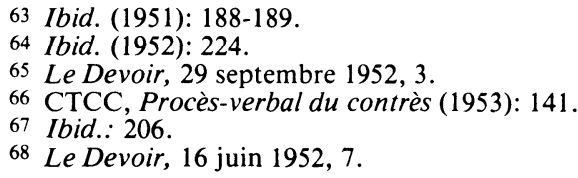


deux réprouvaient le gouvernement Duplessis. C'est l'antisyndicalisme du gouvernement provincial qui rapprocha les deux groupes syndicaux. Leur collaboration débuta en 1948, lors de la formation d'un cartel intersyndical pour faire échec au projet de loi 5, et se continua par la suite contre les lois 60 (1949), 19 et 20 (1954).

La FPTQ participa elle aussi à ces cartels, mais refusa de se joindre à la marche sur Québec organisée pour protester contre les deux derniers projets de loi. Elle estimait que des représentations auprès du gouvernement étaient plus efficaces que des démonstrations de masse, pour obtenir des modifications au projet de loi ${ }^{69}$. Mais les véritables motifs de son refus s'expliquent plutôt par une recrudescence des rivalités intersyndicales chez ses syndicats affiliés et par le sentiment qu'elle avait acquis que la CTCC possédait une mentalité révolutionnaire ${ }^{70}$.

Au contraire, le militantisme de la CTCC plaisait à la FUIQ; elle développa donc le courant de sympathie qui la portait vers elle. Flle annorta son appui moral et matériel aux grévistes de l'amiante, de Dupuis Frères et de Louiseville, tandis que la CTCC faisait de même pour ceux de Noranda en 1953. Tant de rapports unissaient les deux mouvements, que, en 1956, il apparaissait beaucoup plus plausible que la FUIQ se fusionne avec la CTCC qu'avec la FPTQ. Mais ce choix n'appartenait pas aux syndiqués québécois, il relevait de décisions prises aux Etats-Unis entre la FAT et la COI et leur contrepartie canadienne, le CMTC et le CCT.

Des pourparlers de fusion furent entrepris avec la CTCC lorsque les deux centrales canadiennes, le CMTC et le CCT, se fusionnèrent en 1956. Dès septembre 1955, avant même d'être invitée à se joindre à elles, la centrale catholique prit les devants en nommant un comité de neuf membres chargé d'étudier «les meilleurs méthodes pour réaliser l'unité ouvrière complète au Canada ${ }^{71}$. Après avoir rencontré des représentants du Congrès du Travail du Canada (né de la fusion CMTC-CCT), le comité recommanda l'année suivante, au congrès de la CTCC, non pas une fusion, mais une affiliation comme «union nationale» au CTC. La centrale catholique renonçait à son statut de centrale ouvrière nationale pour se transformer en une vaste «one big union» qui garderait ses

\footnotetext{
69 Ibid., 21 janvier 1954, 3; Le Monde Ouvrier (juin 1954): 2.

70 Le Devoir, 11 juin 1951,3 ; 4 oct. 1951 , 3; 16 juin 1952, 7; 29 sept. 1952, 3.

71 CTCC, Procès-verbal du congrès (1955): 163.
} 
syndicats affiliés ${ }^{72}$. Pour un bon nombre des chefs de la CTCC, dont le président, G. Picard, et le secrétaire, J. Marchand, l'unité était une nécessité, car la division affaiblissait les travailleurs ${ }^{73}$. Sans doute aussi, à cette époque où le nationalisme apparaissait suspect, la CTCC sentait-elle le besoin de s'ouvrir vers l'extérieur. Mais on prenait soin d'écarter l'unité organique car la CTCC possédait des caractéristiques qu'on tenait à garder ${ }^{74}$.

Même avec ces réserves, le projet présenté au congrès de 1956 rencontra de l'opposition lorsqu'il fallut le traduire concrètement; il nécessitait en effet, une modification profonde des structures de la centrale et l'élimination de son statut confessionnel ${ }^{75}$. Là-dessus, le comité de l'unité syndicale reçut une majorité plutôt faible des délégués, et le congrès spécial prévu pour juin 1957, qui devait statuer sur l'affiliation, n'eut pas lieu car des tensions apparurent au sein de la centrale. Un groupe de syndiqués repoussait l'affiliation parce que, pensait-il, la cohabitation avec des syndicats internationaux allait s'avérer impossible et qu'elle signifiait l'abandon par la centrale de son statut confessionnel ${ }^{76}$. La question rebondit au congrès régulier de 1957, qui amenda substantiellement le projet d'affiliation du comité d'unité syndicale en durcissant ses exigences envers le CTC. D'après le projet adopté, la CTCC gardait son autonomie quant à son orientation doctrinale, son expansion et ses structures internes, elle conservait ses conseils centraux et restait indépendante de la FTQ, se réservant même le droit de représentation législative au gouvernement du Québec ${ }^{77}$. Même amendé dans un sens aussi favorable à la CTCC, le projet d'affiliation ne recueillit qu'un appui mitigé des délégués (204 à 189), ce qui indique que l'accord était loin d'être réalisé à l'intérieur de la centrale. De plus, les conditions d'affiliation rendaient presque impossible l'accord du CTC.

D'ailleurs, la centrale canadienne avait elle aussi échoué dans sa tentative pour réduire les obstacles à l'affiliation. À son congrès de 1958, l'exécutif de la centrale s'était vu refuser par les délégués de négocier une entente avec la CTCC sans obtenir le consentement de chaque fédération internationale ayant juridiction profes-

72 Ibid. (1956): 238s.

73 Le Travail, 23 mars 1956, 1; CTCC, Procès-verbal du congrès (1964): 14.

74 Le Travail, 14 octobre, 1955, 1.

75 CTCC, Procès-verbal du congrès (1956): $224 \mathrm{~s}$.

76. Le Travail, 4 octobre 1957,$3 ; 5$ octobre 1956,1 .

77 CTCC, Procès-verbal du congrès (1957): 182-195; G. Dion, «La CTCC et au CTCC», Relations industrielles (janvier 1958): 57-61. 
sionnelle concurrente avec les syndicats de la CTCC ${ }^{78}$. La constitution du CTC exigeait, en effet, un tel consentement qui rendait une entente avec la CTCC bien problématique ${ }^{79}$. En définitive, la réponse à l'offre d'affiliation de la CTCC relevait davantage de centres de décision américains que du CTC, malgré toute la bonne volonté qu'il pouvait manifester. Les fédérations internationales avaient mis de côté le principe d'exclusivité juridictionnelle lors de la fusion de la FAT-COI, mais il est douteux qu'elles aient été prêtes à faire de même pour une centrale aussi minuscule que la CTCC à l'échelle du continent nord-américain. Des obstacles presque insurmontables hypothéquaient donc une entente entre les deux centrales: le CTC avait peu de pouvoir sur ses syndicats affiliés, tandis que la CTCC posait des conditions d'affiliation inacceptables.

Les négociations se poursuivirent néanmoins jusqu'en 1961, la CTCC ayant fait un certain nombre de concessions: elle se montrait prête «à encourager l'élimination des organisations et juridictions doubles» et acceptait la fusion de ses conseiis centraux dans les deux années qui suivraient la signature de l'entente ${ }^{80}$. Restait le problème de l'empiétement de juridiction que le CTC estima finalement impossible à surmonter en 1959. Il retira alors son offre d'accorder le statut d'union nationale à la CTCC, ne laissant plus d'autre choix aux syndicats catholiques que de se fusionner avec ceux des fédérations internationales ${ }^{81}$. "L'échafaudage précaire que nous avions dressé, raconte Jean Marchand, s'écrasa comme un édifice dont les fondations cèdent brusquement. ${ }^{82}$ Emportée aussi par le nouveau nationalisme qui s'affirmait au Québec, la CSN mit définitivement un terme à ses pourparlers en 1961 .

Quand on connaît le long passé de rivalité intersyndicale qui a existé entre la CTCC et les syndicats internationaux, il est étonnant de constater que les deux centrales soient venues si près d'une affiliation. Même s'il n'était pas question que la centrale catholique disparaisse comme entité, il n'en reste pas moins que la CTCC

78 Louis-Laurent Hardy, Brève histoire du syndicalisme ouvrier au Canada (Montréal, Ed. de l'Hexagone, 1958), 137. Nous n'avons pu trouver le texte de cette résolution dans les procès-verbaux du congrès de 1958. Les résolutions présentées au comité des résolutions et rejetées ne figurent pas toutes au procès-verbal. J.-M. Bédard en présenta une semblable au congrès de 1962, sans plus de succès (CLC, Report of Proceedings (1962): 76s).

79 CLC, Report of Proceedings (1956): 94-96, 616.

80 CTCC, Procès-verbal du congrès (1959): 104-111.

81 Ibid. (1960): 324.

82 Ibid. (1964): 16. 
avait subi des transformations énormes depuis la guerre pour se rapprocher autant des internationaux. Mis à part le nationalisme, la frontière idéologique qui séparait les deux organisations s'était sérieusement rétrécie au milieu des années 1950. L'humanisme démocratique à l'enseigne duquel logeait la CTCC n'était guère éloigné de l'idéologie des syndicats internationaux. Pas étonnant alors qu'elle se soit rapprochée de ces syndicats et qu'elle ait senti qu'elle avait de moins en moins de bonnes raisons de diviser les travailleurs.

\section{Le gouvernement provincial}

Dans ses rapports avec le gouvernement provincial, la CTCC mérite sa réputation de foyer des forces antiduplessistes. Ses relations avec le gouvernement devinrent tellement tendues que la centrale catholique cessera de soumettre son mémoire annuel au Premier ministre à partir de 1956. Jusqu'à l'avènement de l'Union nationale, les rapports de la CTCC avec les gouvernements libéraux avaient été assez cordiaux, tout comme ceux d'ailleurs des syndicats internationaux. À la grande satisfaction de la CTCC, les libéraux avaient voté deux lois dont elle était l'instigatrice, la loi des syndicats professionnels (1924) et la loi d'extension juridique des conventions collectives (1934). Mais, dès l'arrivée de Duplessis au pouvoir en 1936, la situation se corsa car le gouvernement fit adopter ou modifia plusieurs lois dans un sens défavorable au mouvement syndical. Selon le président de la CTCC à l'époque, A. Charpentier, le gouvernement s'était livré «à un ensemble d'agissements digne du pire attentat politique contre la vie même du syndicalisme ouvrier dans la province de Québec» ${ }^{83}$. L'élection du parti libéral en 1939 apporta un répit de courte durée car l'Union nationale reprenait le pouvoir en 1944.

Ce qui a surtout déplu au mouvement syndical, ce sont les nombreuses législations votées pour restreindre le champ d'activité et le pouvoir de négociation des syndicats. Le gouvernement s'est employé à soustraire le secteur public et para-public à la syndicalisation (fonctionnaires en 1938, employés des municipalités rurales, 1949) et à leur enlever le droit de grève (employés d'institutions charitables, 1939, employés des villes, 1949), le droit d'arbitrage (institutrices rurales, 1946) ou leur accréditation s'ils faisaient grève (loi 20, 1954). Dans une tentative pour substituer l'action

83 Ibid. (1937): 41. 
gouvernementale à celle des parties patronales et syndicales, il s'est donné le pouvoir en 1937 et 1938 d'abroger ou de modifier un décret consenti selon la loi des conventions collectives sans consulter les parties à la convention ${ }^{84}$. La phobie qu'avait Duplessis de l'atelier fermé se traduisit en 1938 par une loi qui prévoyait des amendes à quiconque attentait à la liberté de travail d'un salarié; le projet de code du travail présenté en 1948, puis retiré à la suite des protestations du mouvement syndical, contenait aussi une disposition rendant illégaux l'atelier fermé et la sécurité syndicale ${ }^{85}$. En outre, sous le couvert de la lutte contre le communisme, le gouvernement Duplessis chercha à affaiblir le mouvement syndical en emprisonnant certains organisateurs ou leaders syndicaux, allant même en 1954 (loi 19) jusqu'à priver de la reconnaissance syndicale une organisation qui tolérait comme organisateur ou officier une personne adhérant au communisme ${ }^{86}$.

L'antisyndicalisme de Duplessis se manifesta aussi par son refus d'apporter à la loi des relations ouvrières des modifications qui auraient rendu son application plus juste pour les syindicâts. Votée en 1944, cette loi avait de nombreuses carences que le mouvement syndical voulait corriger. D'après la CTCC, cette loi adoptée pour permettre à tout ouvrier d'adhérer librement à un syndicat aurait dû interdire les syndicats de boutique et accorder une protection efficace contre les congédiements pour activité syndicale ${ }^{87}$. La centrale s'en prit particulièrement à la Commission chargée d'appliquer la loi, qui aurait montré dans son interprétation un préjugé défavorable aux travailleurs. Comme la nomination de la majorité des commissaires était politique ${ }^{88}$, il s'ensuivait que ses décisions reflétaient souvent le point de vue gouvernemental ou patronal. C'est pourquoi la CTCC développa envers la Commission une véritable aversion à partir de 1953.

En outre, Duplessis eut l'occasion de démontrer son antisyndicalisme lors de plusieurs grèves, alors que sa sympathie se dirigeait d'abord vers la partie patronale. Il avait tendance à concevoir

84 J.R. Cardin, L'influence du syndicalisme national catholique sur le droit syndical québécois (Montréal, Les Cahiers de l'institut Social Populaire, 1957): 39-41.

85 Le Travail, février 1948, 8.

86 Ibid., 15 janvier 1954, 2.

87 Ibid., 14 décembre 1951, 4; 20 juin 1952, 1 et 3; 10 février 1956, 1. Concédons à propos de cette loi que Duplessis abaissa en 1945 de 60 à 50 pour cent le nombre de travailleurs requis dans une entreprise pour qu'un syndicat obtienne un certificat de reconnaissance syndicale obligeant l'employeur à négocier un contrat collectif.

88 A. Charpentier, op. cit., $365 \mathrm{~s}$. 
la grève comme un acte d'insubordination et un facteur de désordre, et d'en attribuer la responsabilité aux excès de certains leaders syndicaux ${ }^{89}$. Selon un raisonnement encore bien connu, c'étaient eux qui fomentaient les grèves et manipulaient les ouvriers. D'où les accusations maintes fois répétées de révolutionnaires, de saboteurs et de dictateurs adressées à leur endroit par le Premier ministre. Il soupçonnait les chefs de la CTCC de vouloir sa défaite et d'utiliser la grève pour satisfaire leur rancoeur politique ${ }^{90}$. Que les chefs syndicaux aient voulu sa défaite, c'est certain, son parti pris patronal ne leur laissait guère le choix.

L'antisyndicalisme de Duplessis nous semble originer de deux sources: l'idéologie conservatrice qui l'animait et l'influence de la législation syndicale nord-américaine. Il y a un parallèle intéressant à tracer entre le contenu de la loi Taft-Hartley adoptée aux États-Unis en 1947 et les législations ouvrières du gouvernement de l'Union nationale. Les deux soustraient le secteur public à la syndicalisation, manifestent le même anticommunisme et veulent interdire le principe de l'atelier fermé ${ }^{91}$. Après la guerre, l'idée semble s'être emparée des gouvernements que les concessions faites au mouvement syndical depuis la crise avaient été trop généreuses et qu'il fallait maintenant renverser la vapeur en restreignant sa liberté de manoeuvre. À ce sentiment que Duplessis partageait avec beaucoup de gouvernements, s'ajoute dans son cas une conception paternaliste des relations patronales-ouvrières. Il avait de la difficulté à concevoir que ces deux groupes sociaux puissent entretenir des rapports d'égalité; il pensait plutôt que les travailleurs devaient être placés dans un état de subordination filiale. Sur un mémo, il écrivait en $1938^{92}$ :

Les patrons doivent diriger leur industrie avec justice, intégrité, bonté, mais à la tête. Les ouvriers, à leur place, sachant obéir aux ordres, accomplissant leur tâche de leur mieux. Eux aussi doivent être justes, honnêtes et bons. Les unions, à leur place, doivent protéger leurs membres, mais pas en les persuadant que le patron est un ennemi, et n'intimidant pas ceux qui ne veulent pas être unionistes. 501.

89 Conrad Black, Duplessis, vol. II, le pouvoir (Montréal, Ed. de l'homme, 1977):

90 En collaboration, En grève!..., op. cit., 170.

91 Henry Pelling, Le mouvement ouvrier aux États-Unis (Paris, Seghers, 1965), $215 \mathrm{~s}$.

92. Robert Rumilly, Maurice Duplessis et son temps (Montréal, Fides, 1973), 1: 464. 
Inspirées de tels principes, on comprend que ses législations se soient attirées les foudres du mouvement syndical, particulièrement de la CTCC, laquelle était aux antipodes au point de vue idéologique. Pendant que Duplessis concevait l'entreprise comme une famille, la CTCC réclamait la cogestion et la copropriété.

L'antagonisme entre le gouvernement et la CTCC provoqua une évolution de la centrale à l'égard de l'action politique. Depuis sa fondation, la centrale catholique s'était toujours opposée à l'action politique partisane; d'ailleurs, sa constitution de 1921 le lui interdisait ${ }^{93}$. L'arme politique avait un cachet socialiste qui seyait mal à une centrale née pour faire échec à cette idéologie. Mais après des événements comme la grève de l'amiante et le dépôt des lois 5 et 60 , plusieurs chefs syndicaux s'interrogaient sur la sagesse de cette position. Ne valait-il pas mieux porter la bataille là d'où venaient les coups, c'est-à-dire sur le terrain politique?

Au congrès de 1949, un pas prudent fut fait dans cette direction quand la centrale forma un comité d'action civique (devenu l'année suivante comité d'orientation politique) ayant pour objectif l'éducation politique des travailleurs. On prenait bien soin de préciser que le comité n'appuierait aucun parti politique et que son rôle se cantonnerait à influencer le législateur, à faire l'éducation civique des travailleurs et à orienter «l'opinion publique vers une collaboration des classes ${ }^{94}$. À la veille des élections provinciales de 1952, le comité reçut du Bureau confédéral le mandat de faire connaître le point de vue de la CTCC sur les programmes proposés par les partis politiques et d'éclairer les électeurs sur la valeur des candidats en présence ${ }^{95}$. Au cours de la campagne, le comité diffusa le programme législatif de la centrale et dénonça officiellement quatre candidats de l'Union nationale pour leur hostilité à l'endroit de la $\mathrm{CTCC}^{96}$. Ce dernier geste, que certains délégués au congrès de 1952 interprétèrent comme de la partisanerie politique, provoqua un long débat qui se termina par une résolution confirmant les orientations tracées par le Bureau confédéral ${ }^{97}$. Tout en

93 CTCC, Constitution et Règlements de la Confédération des Travailleurs Catholiques du Canada (Québec, Imp. L’Action Sociale Ltée, 1923), 25. Pour un historique de l'action politique de la CTCC, voir Guy Lortie, «Évolution de l'action politique de la CSN», Relations industrielles, 22, 4 (octobre 1967): 532-554.

94 CTCC, Procès-verbaux du congrès (1949): 95.

95 Ibid. (1952): 80.

96 Ibid. (1951): 81.

97 Ibid. (1952): 172-178. 
repoussant l'action politique partisane, la majorité comprenait la nécessité de gestes politiques concrets.

Pour certains, la situation politique au Québec commandait de recourir à des moyens d'action plus vigoureux. Un permanent libéré exclusivement pour l'éducation politique fut finalement désigné; et l'idée d'appuyer, en collaboration avec les groupements intéressés, des candidats soucieux du bien commun fut approuvée au congrès de $1954^{98}$. Un pas de plus fut accompli en 1959 quand le congrès amenda la constitution de façon à permettre aux corps affiliés de prendre les attitudes qu'ils jugeraient appropriées en matière politique, en autant qu'elles ne venaient pas «à l'encontre des intérêts généraux du mouvement ${ }^{99}$. Cependant la centrale ne se compromit pas davantage car elle rencontrait de fortes résistances chez ses membres.

À la fin des années 1950, un certain nombre d'événements amenèrent la CTCC à s'interroger sur l'opportunité de faire le saut en politique active. À son congrès de 1958, le Congrès du Travail du Canada invitait tous les groupes syndicaux à s'entendre pour former un nouveau parti politique populaire. En réponse à cet appel, le Bureau confédéral forma un comité et lui donna ce mandat assez vague de «discuter des objectifs politiques du mouvement ouvrier ${ }^{100}$. Mais au congrès, tenu quelques mois plus tard, deux résolutions soumises à l'effet d'étudier la possibilité de former un parti politique canadien furent rejetées par le Comité des résolutions, et la centrale réaffirma sa volonté de garder son indépendance envers tous les partis politiques ${ }^{101}$. Quelques dirigeants de la centrale, G. Picard, M. Chartrand et P. Vadeboncoeur, jouèrent un rôle clé dans la formation du NPD au Québec, sans toutefois vaincre les réserves des militants de la CTCC.

Il est remarquable de constater que la centrale catholique avait de fortes réticences à dépasser l'action politique éducative pour déboucher même sur un appui indirect à un parti politique. Il existe une opinion bien ancrée chez les syndiqués québécois qui veut qu'on dissocie l'action syndicale de l'action politique partisane. Dans la plus pure tradition "gompérienne», la finalité du syndicalisme, pense-t-on, est purement économique; en politique,

98 Ibid. (1954): 178-180.

99 Ibid. (1959): 263; La Gazette du Travail (1959): 1318.

100 Ibid. (1959): 71.

101 Ibid. (1959): 262. La Gazette du Travail (1958): 1410. 
le travailleur agit comme citoyen et non comme syndiqué. Les syndicats internationaux n'ont pas échappé à ce sentiment dominant au Québec; leur appui au CCF-NPD s'est révélé plutôt timide et, de toute façon, leur mot d'ordre n'a pas été suivi par leurs membres.

Contre ce sentiment, la CTCC après la guerre a marqué le pas en ajoutant à la méthode d'action politique, dite de pression ou d'influence auprès des gouvernements, l'action politique éducative. Cette évolution s'explique par le militantisme accru de la centrale et surtout par l'antisyndicalisme de Duplessis, qui ne laissa pas de répit aux dirigeants de la CTCC. Plusieurs d'entre eux auraient bien voulu remettre au premier ministre sur le terrain politique les coups qu'il leur portait, mais la majorité n'était pas encore prête à impliquer la centrale dans la politique partisane.

Au terme de cette étude, il ressort que la CTCC a subi des transformations profondes après la guerre. Elle a audinduniné l'idéal corporatiste et son caractère confessionnel. Sur un autre plan, elle a accentué son militantisme à l'égard du patronat et du gouvernement.

Il convient en conclusion de se demander quels sont les facteurs sous-jacents à cette mutation radicale de la centrale catholique. En réponse à cette question, Louis-Marie Tremblay, dans son volume sur les idéologies de la CSN et de la FTQ, retenait comme facteurs principaux d'explication l'évolution de l'économie québécoise, la transformation de la pensée sociale canadiennefrançaise et le renouveau du leadership syndical ${ }^{102}$. Il est toujours hasardeux d'imputer aux orientations idéologiques ou aux leaders syndicaux des changements qui peuvent originer de la base syndicale elle-même. J'en suis conscient, mais à défaut de monographies syndicales sur cette période, je suis obligé de me rabattre sur des explications aussi générales. Parmi les raisons amenées par L.-M. Tremblay pour expliquer le renouveau de la CTCC, je ne crois pas que la vague d'industrialisation qui a touché le Québec après la guerre soit un facteur décisif. Les syndicats internationaux affiliés à la FAT, largement majoritaires au Québec, n'ont pas attendu l'après-guerre pour afficher un militantisme qui, d'ailleurs, ne s'accentue pas dans les années 1950, sauf pour les quel-

102 L.-M. Tremblay, op. cit., 27-29. 
ques syndicats affiliés à la FUIQ ${ }^{103}$. De même, l'arrivée de nouveaux leaders syndicaux à la tête de la CTCC ne me semble pas responsable en soi de changements décisifs; ce sont plutôt les idées qu'ils portent qui sont génératrices de renouveau.

Plus significatives donc m'apparaissent les transformations idéologiques subies par la centrale. La jeune génération de clercs et de laïcs catholiques d'après-guerre rejette le corporatisme trop associé, dans son esprit, au fascisme pour être une solution de rechange valable au capitalisme. Au Québec comme ailleurs, la victoire des démocraties libérales lors du dernier conflit mondial a porté un dur coup aux idéologies de droite, d'où le corporatisme était issu. Cette victoire a fortement ébranlé l'idéologie cléricoconservatrice que l'Église diffusait au Québec depuis le XIXe siècle. L'esprit plus libéral qui gagne ses éléments les plus jeunes après la guerre se traduit par une séparation plus claire entre le temporel et le spirituel et signifiera l'abandon de la confessionnalité par la CTCC. Dans le domaine des relations de travail, les jeunes clercs et laïcs catholiques sont à la recherche de voies alternatives au corporatisme et au capitalisme; ils prôneront la réforme de l'entreprise, puis un humanisme démocratique. Cette recherche créera une dynamique du changement, propre à insuffler à la CTCC un militantisme accru. L'abandon du corporatisme m'apparaît avoir créé un climat favorable à l'adoption de nouvelles idéologies et au recours à des pratiques syndicales plus radicales.

103 En 1951 et 1955 , le COI possédait respectivement 53 et 112 syndicats affiliés au Québec, contre 331 et 416 pour la FAT (Canada, Ministère du travail, Organisation des travailleurs au Canada (1951): 24-69, (1955): 30-104. 